\title{
Givex
} CASE BASED DISCUSSIONS

\section{Exercise-induced haemoptysis as a rare presentation of a rare lung disease}

\author{
Andrew D Mihalek, ${ }^{1,2,3}$ Carissa Haney, ${ }^{4}$ Maria Merino, ${ }^{5}$ Sinchita Roy-Chowdhuri, ${ }^{5,6}$ \\ Joel Moss, ${ }^{1}$ Kenneth N Olivier ${ }^{1,4}$
}

${ }^{1}$ Cardiovascular and Pulmonary Branch, National Heart, Lung, and Blood Institute (NHLBI), National Institutes of Health (NIH), Bethesda, Maryland, USA

${ }^{2}$ Division of Pulmonary and

Critical Care Medicine, Department of Internal Medicine, University of Virginia, Charlottesville, Virginia, USA

${ }^{3}$ Lovelace Respiratory Research Institute, Albuquerque, New

Mexico, USA

${ }^{4}$ Laboratory of Clinical

Infectious Diseases, National Institute of Allergy and Infectious Diseases (NIAID), National Institutes of Health (NIH), Bethesda, Maryland, USA

${ }^{5}$ Department of Pathology, National Cancer Institute (NCI), National Institutes of Health (NIH), Bethesda, Maryland, USA

${ }^{6}$ Department of Pathology, University of Texas MD Anderson Cancer Center, Houston, Texas, USA

\section{Correspondence to}

Dr Kenneth N Olivier,

Cardiovascular and Pulmonary Branch, NHLBI, 10 Center

Drive, MSC 1454, Bldg

10-CRC, Rm 3130, Bethesda

MD 20892, USA;

olivierk@nhlbi.nih.gov

Received 3 November 2015

Accepted 2 May 2016

Published Online First

7 June 2016

\section{CrossMark}

To cite: Mihalek $A D$, Haney C, Merino $M$, et al. Thorax 2016;71:865-868.

\section{ABSTRACT}

Amyloid primarily affecting the lungs is a seldom seen clinical entity. This case discusses the work-up of a patient presenting with exercise-induced haemoptysis and diffuse cystic lung disease on radiographic imaging. The common clinical and radiographic findings of diffuse cystic lung diseases as well as a brief overview of pulmonary amyloid are presented.

ADM (pulmonary fellow): A young patient without prior medical history presented to the National Institutes of Health (NIH) in Bethesda, Maryland, USA for evaluation of a 4-year history of exercise-induced haemoptysis. Originally from Africa, the patient was 22 years old when they experienced an episode of haemoptysis after swimming approximately two miles. The haemoptysis was self-limited and the patient estimated expectorating approximately $100-200 \mathrm{~mL}$ of frank blood. Over the course of the next 2 years, the patient experienced similar episodes, each time occurring after a period of swimming. The patient denied chest pain, shortness of breath, presyncopal symptoms and constitutional symptoms. Prior to this, the patient enjoyed a variety of athletic activities without issue.

KNO (staff pulmonologist): The chief complaint of the patient was the occurrence of frank haemoptysis following vigorous exercise. The fact that symptoms occurred while swimming is an intriguing aspect of this patient's presentation and calls to mind the clinical entity known as swimminginduced pulmonary oedema (SIPO). The existence of pulmonary oedema with land-based exercise in individuals without underlying left ventricular disease is controversial and limited to case reports. ${ }^{1}{ }^{2}$ However, SIPE is a well-documented occurrence in military trainees, open-water swimmers and divers. Exercise leads to increases in cardiac output resulting in rises in the pressure gradient across the pulmonary capillary bed. The overall increase in hydrostatic forces across the pulmonary capillary bed is generally well tolerated as a result of competing oncotic pressures and the fact that cells composing the alveolar epithelium share a singular, fused basement membrane. When compared with sedentary subjects, bronchoalveolar lavage fluid from elite athletes undergoing intense exercise have higher concentrations of red blood cells, total protein and albumin, providing evidence of compromised blood-gas barrier integrity at peak exercise. $^{3}$ Immersion and exercise in water, particularly cold water, causes a centralisation of blood flow and augments the increases in pulmonary artery pressure seen during exercise. SIPE is thought to arise in susceptible individuals who experience these physiological changes during swimming. Though presenting in similar fashion to heart failure, patients with SIPE demonstrate normal serum levels of brain natriuretic peptide and lack echocardiographic evidence of heart failure. The incidence and prevalence of SIPE are unknown. One in five individuals with SIPE will experience reoccurrence of their symptoms when challenged and as many as $56 \%$ of patients with SIPE will experience episodes of frank haemoptysis. ${ }^{4}$

ADM: The patient was evaluated by a pulmonologist in Africa and prescribed two courses of an unknown antibiotic. Despite these interventions, the patient continued to experience episodes of haemoptysis while swimming. Sputum cultures taken at numerous times were negative for bacterial, fungal and acid-fast organisms. The patient was eventually referred to the NIH and evaluated on both pulmonary training and host defense research protocols. At the NIH, the patient continued to report occasional episodes of haemoptysis with rigorous exercise but otherwise denied shortness of breath, chest pain, arthralgias, myalgias, rashes or constitutional symptoms. Now an immigrant to the USA, the patient worked at a local restaurant. The patient reported casual tobacco use $(<500$ lifetime cigarettes) but had refrained from tobacco consumption in the year prior to seeing us. The patient denied illicit drug use. The patient's sibling had been treated for TB during their youth, but the patient otherwise denied other familial maladies.

CH (nurse practitioner): At presentation to the $\mathrm{NIH}$, the patient was afebrile, vital signs were normal, and with the exception of poor dentition, the physical examination was unremarkable. Basic blood work, an infectious work-up (including induced sputum), a transthoracic echocardiogram and a bronchoscopy were without significant findings. A purified protein derivative test was negative and alpha-1 antitrypsin serum levels were within normal limits. The patient had normal pulmonary function tests including a normal diffusion capacity, did not demonstrate exertional hypoxaemia or haemoptysis, and had a peak oxygen consumption of $85 \%$ of predicted during a cardiopulmonary exercise test (CPET). Radiographic studies obtained at the $\mathrm{NIH}$ are presented in figure $1 \mathrm{~A}, \mathrm{~B}$. 
A

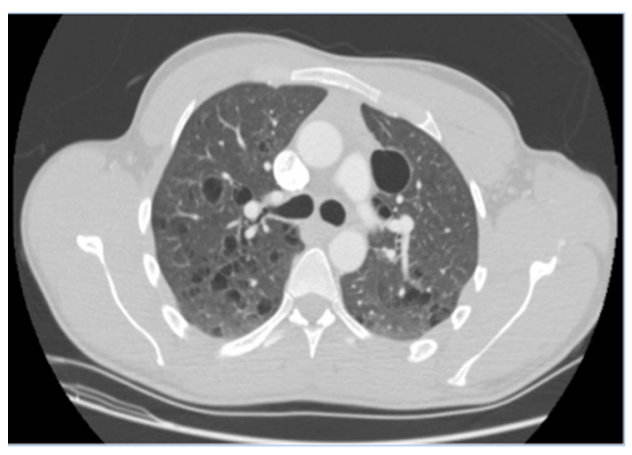

C

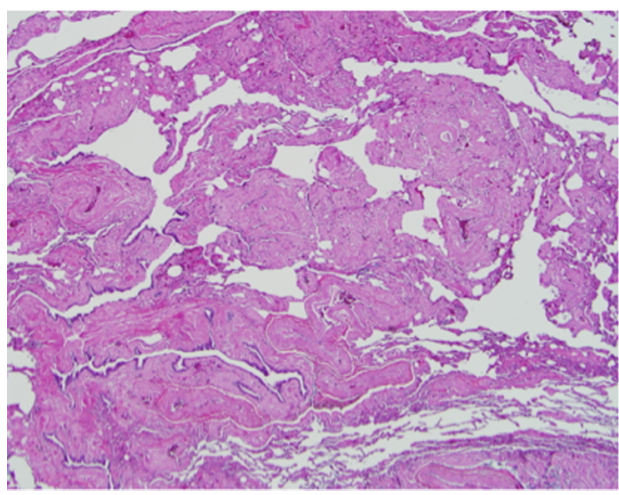

B

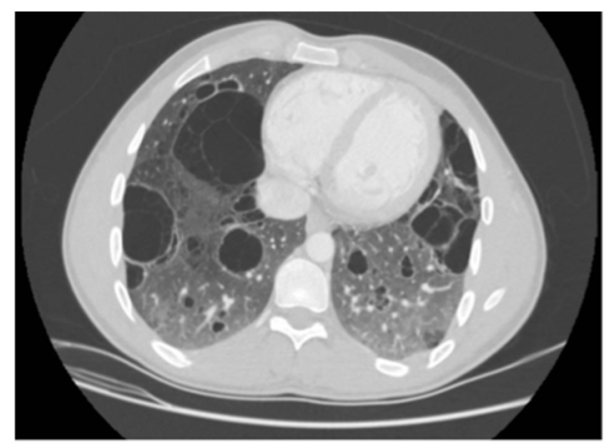

D

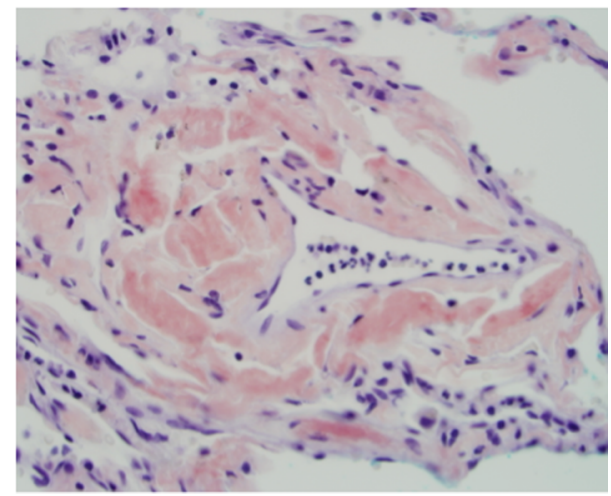

Figure 1 (A and B) Representative non-contrast computed tomographic scan of the chest highlighting the presence of diffuse, thin-walled cysts in a patient presenting with exercise-induced haemoptysis. (C) H\&E stain $(\times 150)$ from the patient's lung biopsy demonstrating diffuse deposition of eosinophilic amorphous material in the lung parenchyma. (D) At a higher power $(\times 200)$, Congo red staining demonstrates that amyloid deposition is particularly striking surrounding the pulmonary vascular bed.

JM (staff pulmonologist): The presence of diffuse, thin-walled cysts is a striking feature of these radiographic images. Cysts are identified by areas of lucency that are surrounded by tissue and are differentiated from cavities by the appearance of thin $(<2 \mathrm{~mm})$ walls of tissue. The appearance of diffuse (implying the involvement of all lobes) cystic lung changes is characteristic of a limited number of diseases (see table 1) that primarily involve the lung, are associated with other systemic diseases, or have genetic origins. ${ }^{56}$

Of the noted diseases, lymphangioleiomyomatosis (LAM) is the most commonly encountered cystic disease. LAM is thought to arise from dysregulation of the mechanistic target of rapamycin signalling pathway resulting in the proliferation of abnormal, smooth muscle-like cells within the walls of the cysts and pulmonary interstitium. Presence of the tuberous sclerosis complex (TSC), an autosomal dominant genetic disorder that occurs in 1 of 6000 live births, increases the likelihood of developing LAM. However, the exact prevalence of LAM in women with TSC is difficult to appreciate given the observation that cyst development tends to occur in an age-dependent manner. Hence, the prevalence of cystic disease in TSC women is thought to be between $26 \%$ and $38 \%$, but has also been seen in as many as $80 \%$ of these patients. ${ }^{9}$ Though male patients with LAM have been documented, this is exceedingly rare and case reportable.

Pulmonary Langerhans cell histiocytosis (LCH) is also one of the more commonly encountered diseases listed. Though the true incidence and prevalence of pulmonary $\mathrm{LCH}$ is unknown, it is almost exclusive to patients with a smoking history and presents with a peak incidence between 20 and 40 years of age. ${ }^{10}$ Pulmonary LCH presents with nodules and cysts in the upper lobes of the lungs. The cavitation of these nodules is thought to lead to the development of cysts. The lack of nodules, the involvement of cysts in the lung bases (not shown), and the patient's unimpressive smoking history make pulmonary $\mathrm{LCH}$ an unlikely diagnosis in this patient. Most of the diseases presented in table 1 are chronic processes that progress slowly over time. Identifying the correct aetiology of diffuse cystic changes is important for prognostic and treatment purposes; however, deriving a clear diagnosis often requires a biopsy.

ADM: Based on the persistence of symptoms and an abnormal CT scan, the patient was referred for a video-assisted thoracoscopic surgery. Wedge biopsies were taken from both the left upper and lower lobes.

SR-C (pathology fellow) and MM (staff pathologist): Results from the patient's surgical biopsy demonstrate interstitial and perivascular deposition of eosinophilic and amorphous hyaline material (figure 1C, D). Samples were positive for Congo red stain and were typified by the apple-green birefringence pathognomonic of amyloid deposition. The biopsy was consistent with a diagnosis of pulmonary amyloid. Samples were not available for further amyloid subtyping by either immunohistochemistry or mass spectroscopy-based proteomics.

JM: Amyloidosis involves the deposition of protein-like substances assembled into bundles as a result of dysfunctional polypeptide folding. Amyloid production and deposition most commonly occurs as two distinct disease processes. The deposition of immunoglobulin light-chain derivative amyloid (AL amyloidosis) is the most common cause of amyloidosis and can affect a single organ such as the skin, eyes and lung. AL amyloidosis is a consequence of either a plasma cell dyscrasia (such as multiple myeloma or monoclonal gammopathy of 
Table 1 Categorisation and features of diffuse cystic lung diseases 578

\begin{tabular}{|c|c|c|}
\hline & Clinical features & Characteristic CT findings \\
\hline \multicolumn{3}{|c|}{ Cystic lesions primarily of the lung } \\
\hline $\begin{array}{l}\text { Light-chain derived (AL) } \\
\text { pulmonary amyloidosis }\end{array}$ & $\begin{array}{l}\text { Associated with blood dyscrasias and lymphoproliferative } \\
\text { disorders }\end{array}$ & $\begin{array}{l}\text { Cysts of diverse sizes and shapes; seen more commonly with septal } \\
\text { thickening, irregular nodularity and tracheobronchial lesions }\end{array}$ \\
\hline LAM & $\begin{array}{l}\text { Associated with pneumothorax, chylous effusions/ascites, renal } \\
\text { angiomyolipomas and/or tuberous sclerosis complex }\end{array}$ & $\begin{array}{l}\text { Round, uniform cysts intermittently dispersed throughout normal lung } \\
\text { parenchyma }\end{array}$ \\
\hline Langerhans cell histiocytosis & Almost exclusively in smokers & Associated with centrilobular nodules; classically spares costophrenic angle \\
\hline Sporadic cystic lung disease & $\begin{array}{l}\text { Associated with small airways disease, hypothesised to be a } \\
\text { consequence of chronic small airway injury }\end{array}$ & Cysts of diverse sizes and shapes; associated with evidence of bronchiolitis \\
\hline Follicular bronchiolitis & $\begin{array}{l}\text { Thought to be on similar pathological spectrums, follicular } \\
\text { bronchiolitis and LIP are almost clinically indistinguishable; }\end{array}$ & $\begin{array}{l}\text { Cysts of diverse sizes and shapes; associated peribronchial distribution with } \\
\text { parenchymal nodularity and tree-in-bud like appearances }\end{array}$ \\
\hline LIP & can be associated with connective tissue diseases & $\begin{array}{l}\text { Cysts of diverse sizes and shapes; associated with perivascular distribution } \\
\text { and ground-glass opacities }\end{array}$ \\
\hline \multicolumn{3}{|c|}{ Cystic lesions secondary to other diseases } \\
\hline $\begin{array}{l}\text { Serum amyloid } A(A A) \\
\text { pulmonary amyloidosis }\end{array}$ & $\begin{array}{l}\text { Generally well tolerated and associated with diseases of } \\
\text { chronic inflammation }\end{array}$ & Similar to primary pulmonary amyloidosis \\
\hline Cystic metastasis & $\begin{array}{l}\text { Reported in a diversity of cancers including colon cancer, } \\
\text { bladder cancer, sarcoma and stromal tumours }\end{array}$ & Cysts of diverse sizes and shapes; cysts can be filled with either fluid or air \\
\hline HIV & $\begin{array}{l}\text { Most profound in patients who smoke; unclear relation to } \\
\text { infections }\end{array}$ & Large, irregularly shaped cysts with emphysematous appearances \\
\hline \multicolumn{3}{|c|}{ Cystic lesions with genetic origins } \\
\hline Tuberous sclerosis complex & Linked to mutations in the TSC1 or TSC2 gene & Radiographically similar to LAM \\
\hline Birt-Hogg-Dubé syndrome & $\begin{array}{l}\text { Linked to mutations in the } F L C N \text { gene; familial history of } \\
\text { pneumothorax }\end{array}$ & $\begin{array}{l}\text { Irregularly sized and shaped cysts; typically subpleural most predominant in } \\
\text { lower lobes }\end{array}$ \\
\hline Cystic fibrosis & $\begin{array}{l}\text { Linked to mutations to the CFTR gene; associated with } \\
\text { recurrent pulmonary infections, pancreatic insufficiency and } \\
\text { nutritional deficiencies }\end{array}$ & $\begin{array}{l}\text { Cysts of varying sizes favouring central, apical presentations associated with } \\
\text { bronchiectasis }\end{array}$ \\
\hline
\end{tabular}

undetermined significance) or a lymphoproliferative disease (such as Waldenström's macroglobulinaemia or bronchial mucosa-associated lymphoid tissue). Sjögren's syndrome, a disease increasingly recognised as a lymphoproliferative disorder, is also associated with AL pulmonary amyloidosis. ${ }^{11}$ Amyloid production can also accompany inflammatory diseases and results in accumulation of the acute-phase reactant amyloid A (AA amyloidosis). Though AA amyloidosis can present with pulmonary involvement, it is rarely symptomatic and treatment plans are most often defined by the presence of amyloid in other target organs such as the heart, liver and kidneys. AA amyloid isolated to the lung is a rare occurrence and prognosis is unknown. By comparison, AL amyloid in the lung has a poor prognosis (median survival thought to be under a year) and is poorly tolerated. AL amyloidosis may present as septal, pleural, nodular, tracheobronchial or cystic disease. Treatment of $\mathrm{AL}$ amyloidosis primarily involves addressing the underlying disease process driving amyloid production (though surgical debulking may improve tracheobronchial depositions that hinder airflow). ${ }^{12} 13$

KNO: It is worth noting again that we were unable to observe any haemoptysis in our patient during CPET administration. However, similar to reports made elsewhere, ${ }^{14}$ the biopsy results from our patient demonstrate the localisation of amyloid along the pulmonary vascular bed. We hypothesise that the presence of these amyloid deposits compromised the integrity of the capillary bed and made the patient susceptible to SIPE by the previously described mechanisms.

JM: AL amyloid manifesting as pulmonary cystic disease is extremely rare and poorly understood; even less is known of AA amyloid in the lung. Case reports examining patients with cystic disease from AL amyloidosis associated with Sjögren's syndrome imply multiple mechanisms for cyst formation perhaps involving airways narrowed or destroyed by inflammatory cells. ${ }^{11}$ Cystic lung disease caused by amyloid is often accompanied by calcified nodules (which this patient did not have). ${ }^{14}$ Because the differences in prognosis and treatment of $\mathrm{AA}$ and AL amyloidosis vary greatly, a thorough investigation to identify underlying systemic diseases is warranted.

$\mathrm{CH}$ : The patient recovered from the biopsy without issue. A work-up was undertaken to identify conditions associated with amyloidosis. A serological panel for collagen vascular disease and both serum and urine electrophoresis studies were unremarkable. A salivary biopsy, undertaken because of the patient's poor dentition, was negative for evidence of Sjögren's syndrome. Given the patient's young age, their intact haematological cell lines, and normal pulmonary function, it was estimated that the patient was suffering from AA amyloidosis. A bone marrow biopsy was not offered and a conservative treatment plan with regular monitoring was employed. Yearly CT scans, blood work, pulmonary function tests and CPET evaluations for the next 4 years were without evidence of worsening symptoms, disease progression or functional impairment. The patient was eventually lost to follow-up.

Funding Supported in part by the Intramural Research Program, National Institutes of Health, National Heart, Lung, and Blood Institute and National Institute of Allergy and Infectious Diseases.

Competing interests None declared.

Provenance and peer review Not commissioned; externally peer reviewed.

\section{REFERENCES}

1 Bates ML, Farrell ET, Eldridge MW. The curious question of exercise-induced pulmonary edema. Pulm Med 2011;2011:361931.

2 Ghio AJ, Ghio C, Bassett M. Exercise-induced pulmonary hemorrhage after running a marathon. Lung 2006;184:331-3. 


\section{Chest clinic}

3 Ludwig B, Mahon R, Parrish J, et al. Pulmonary edema in combat swimmers: clinical and bronchoalveolar lavage description demonstrating stress failure of the pulmonary capillaries. Undersea Hyperb Med 2004;31:C42.

4 Adir Y, Shupak A, Gil A, et al. Swimming-induced pulmonary edema: clinical presentation and serial lung function. Chest 2004;126:394-9.

5 Clarke BE. Cystic lung disease. J Clin Pathol 2013:66:904-8.

6 Seaman DM, Meyer CA, Gilman MD, et al. Diffuse cystic lung disease at high-resolution CT. AJR Am J Roentgenol 2011;196:1305-11.

7 Gupta N, Vassallo R, Wikenheiser-Brokamp KA, et al. Diffuse cystic lung disease. Part I. Am J Respir Crit Care Med 2015;191:1354-66.

8 Gupta N, Vassallo R, Wikenheiser-Brokamp KA, et al. Diffuse cystic lung disease. Part II. Am J Respir Crit Care Med 2015;192:17-29.
9 Taveira-DaSilva AM, Moss J. Clinical features, epidemiology, and therapy of lymphangioleiomyomatosis. Clin Epidemiol 2015;7:249-57.

10 Tazi A. Adult pulmonary Langerhans' cell histiocytosis. Eur Respir J 2006;27:1272-85

11 Baqir M, Kluka EM, Aubry MC, et al. Amyloid-associated cystic lung disease in primary Sjögren's syndrome. Respir Med 2013;107:616-21.

12 Berk JL, O'Regan A, Skinner M. Pulmonary and tracheobronchial amyloidosis. Semin Respir Crit Care Med 2002;23:155-65.

13 de Almeida RR, Zanetti G, Pereira ESJL, et al. Respiratory tract amyloidosis. State-of-the-art review with a focus on pulmonary involvement. Lung 2015;193:875-83.

14 Zamora AC, White DB, Sykes AG, et al. Amyloid-associated cystic lung disease. Chest 2016;149:1223-33. 